\title{
Review Article \\ Organ-Protective Effects and the Underlying Mechanism of Dexmedetomidine
}

\author{
Naren Bao $\mathbb{D}$ and Bing Tang $\mathbb{D}$ \\ Department of Anesthesiology, The First Hospital of China Medical University, 155 Nanjing North Street, Shenyang, China \\ Correspondence should be addressed to Bing Tang; btang@cmu.edu.cn
}

Received 3 March 2020; Revised 13 April 2020; Accepted 16 April 2020; Published 9 May 2020

Guest Editor: Xiaolu Jin

Copyright (C) 2020 Naren Bao and Bing Tang. This is an open access article distributed under the Creative Commons Attribution License, which permits unrestricted use, distribution, and reproduction in any medium, provided the original work is properly cited.

\begin{abstract}
Dexmedetomidine (DEX) is a highly selective $\alpha 2$ adrenergic receptor ( $\alpha 2 \mathrm{AR})$ agonist currently used in clinical settings. Because DEX has dose-dependent advantages of sedation, analgesia, antianxiety, inhibition of sympathetic nervous system activity, cardiovascular stabilization, and significant reduction of postoperative delirium and agitation, but does not produce respiratory depression and agitation, it is widely used in clinical anesthesia and ICU departments. In recent years, much clinical study and basic research has confirmed that DEX has a protective effect on a variety of organs, including the nervous system, heart, lungs, kidneys, liver, and small intestine. It acts by reducing the inflammatory response in these organs, activating antiapoptotic signaling pathways which protect cells from damage. Therefore, based on wide clinical application and safety, DEX may become a promising clinical multiorgan protection drug in the future. In this article, we review the physiological effects related to organ protection in $\alpha 2 \mathrm{AR}$ agonists along with the organ-protective effects and mechanisms of DEX to understand their combined application value.
\end{abstract}

\section{Introduction}

Dexmedetomidine (DEX) is a highly selective $\alpha 2$ adrenergic receptor $(\alpha 2 \mathrm{AR})$ agonist currently used in the clinic which binds in ratio to the adrenergic receptor $-\alpha 2: \alpha 1$ is close to 1620 : 1. In 1999, the United States Drug and Food Administration (FDA) approved DEX for sedation and analgesia in short-term intensive care [1]. In 2008, DEX was approved by the FDA for pre- and intraoperative sedation in nontracheal intubation patients. It was then approved, in 2009, for sedating patients under general anesthesia, endotracheal intubation, and mechanical ventilation. Because DEX has the dose-dependent advantages of sedation, analgesia, antianxiety, inhibition of sympathetic nervous system activity, cardiovascular stabilization, and significant reduction of postoperative delirium and agitation, but does not produce respiratory depression and agitation, it is widely used in clinical anesthesia and the ICU.
In recent years, much clinical and basic research has confirmed that DEX has a protective effect on a variety of organs, including the nervous system, heart, lungs, kidneys, liver, and small intestine. It reduces inflammatory response, activating antiapoptotic signaling pathways which protect cells from damage. Therefore, based on wide clinical application and safety, DEX may become a promising clinical multiorgan protection drug in the future. In this article, we review the physiological effects related to organ protection in $\alpha 2 \mathrm{AR}$ agonists along with the organ-protective effects and mechanisms of DEX to understand their combined application value.

\section{Main Physiological Effects and Molecular Mechanisms Related to Organ Protection by $\alpha 2 A R$ Agonists}

In the body, $\alpha 2$ adrenoceptors ( $\alpha 2 \mathrm{AR}$ ) have three subtypes including $\alpha 2 \mathrm{~A}, \alpha 2 \mathrm{~B}$, and $\alpha 2 \mathrm{C}$. These are widely distributed 
in the central and peripheral nervous systems, autonomic ganglia, vital organs, and blood vessels $[2,3]$. The main physiological effects and molecular mechanisms involved in organ protection by $\alpha 2 \mathrm{ARs}$ are summarized as follows.

2.1. Sedative and Hypnotic Effects. Central norepinephrine has the effect of maintaining brain arousal. $\alpha 2 \mathrm{AR}$ agonists activate the $\alpha 2 \mathrm{AR}$ located in the locus coeruleus of the brainstem, producing an inhibition of adenylate cyclase activity and reduction of cyclic adenosine monophosphate. They also promote the outflow of potassium ions and inhibit the inflow of calcium ions in nerve endings, which leads to hyperpolarization of the cell membrane. This inhibits the discharge of neurons in the locus coeruleus and the release of norepinephrine. All these effects produce sedation and hypnosis [4].

2.2. Analgesic Effect. $\alpha 2 \mathrm{AR}$ agonists produce analgesic effects in multiple sites. In the brain, they bind to the $\alpha 2 \mathrm{AR}$ in the brainstem locus coeruleus to stop the transmission of pain signals. In the spinal cord, they activate $\alpha 2 \mathrm{AR}$ in the presynaptic membranes of neurons in the posterior horn and the postsynaptic membranes of intermediate neurons to open the potassium ion channel. Thus, promotion of potassium ion outflow and inhibition of calcium ion inflow lead to the cell membrane's hyperpolarization, ultimately inhibiting the transmission of pain signals to the brain. In the periphery, they inhibit the activation of nociceptive neurons by $\mathrm{A} \delta$ and C-type nerve fibers along with the release of the nociceptive neurotransmitter $\mathrm{P}$ and other nociceptive peptides [5].

2.3. Antisympathetic Effects. $\alpha 2 \mathrm{AR}$ agonists can activate the $\alpha 2 \mathrm{AR}$ of the brainstem locus coeruleus to inhibit the release of norepinephrine through negative feedback. This can inhibit sympathetic nerve excitability, reduce plasma catecholamine concentration, stabilize hemodynamics, reduce blood pressure and heart rate, and produce antisympathetic effects [6].

\subsection{Affects $G$ Protein and Intracellular Signaling. $\alpha 2 \mathrm{ARs}$} belong to the G protein-coupled receptors (GPCRs) across the cell membrane and have the general biological properties of G protein. GPCRs are extensively expressed throughout the body. When they are activated, GPCRs interact with their cognate $G$ protein. They transmit extracellular stimulation into the cell by amplifying transduction and triggering a series of intracellular responses. $\mathrm{G}$ proteins, being heterotrimeric, are composed of three subunits: $\alpha, \beta$, and $\gamma$. The enormous quantities of $\mathrm{G}$ protein families-e.g., $18 \alpha$ subunits, $12 \beta$ subunits, and $5 \gamma$ subunits - indicate that the signal transduction pathway triggered by GPCR is very intricate [7]. The $\mathrm{G}$ proteins activating adenylate cyclase are called Gs, and other inhibitors are called Gi. $\alpha 2 \mathrm{ARs}$ are classic inhibitory GPCRs $[8,9]$ which couple to the pertussis toxin-sensitive inhibitory $\mathrm{G}$ protein $(\mathrm{G} \alpha \mathrm{i})$ and produce a multifarious biological effect in multiple organs. However, the molecular mechanism after $\alpha 2 \mathrm{AR}$ receptor activation is not clear. At present, some believe that $\alpha 2 \mathrm{AR}$ directly inhibits the AC-cAMP-PKA pathway through G $\alpha$ i proteins and the phosphorylation of the CAMP response element-binding protein (CREB) $[10,11]$. Additionally, the separation of the $\mathrm{G} \beta \gamma$ subunit from $\mathrm{G} \alpha \mathrm{i}$ activates the PI3K/Akt pathway, phospholipase C, ERK, etc. [9, 12-14]. Both of the pathways mentioned above ultimately lead to the activation of NF- $\kappa \mathrm{B}$ [9]. However, some others point out that $\alpha 2$ ARs can couple both physically and functionally to Gs, producing diametrically opposite physiological effects under high concentrations of agonist and high receptor expression. Moreover, this Gs coupling is subtype-selective $[15,16]$. In fact, it seems to be inconsistent with the physiological effects of $\alpha 2 \mathrm{AR}$ as a GPCR; most clinical researchers show that DEX produces anti-inflammatory and organ-protective effects by inhibiting NF- $\kappa$ B. Based on the results of the above discussion, we speculate that DEX may couple with Gs to stimulate AC-cAMP-PKA pathways or affect PI3K/Akt pathways, etc., thus inhibiting NF- $\kappa \mathrm{B}$ in situations such as inflammation and ischemia, or applications within a certain concentration range.

2.5. Inhibits the Activity of Adenylyl Cyclase (AC). Cyclic adenosine monophosphate (cAMP), an important intracellular functional regulator, regulates the target protein activity by controlling its phosphorylation. AC is the main factor in regulating the amount of intracellular CAMP, and a change in its activity directly leads to cAMP synthesis change. Studies have pointed out that agonistic $\alpha 2 \mathrm{AR}$ has the ability to inhibit AC activity in order to reduce intracellular cAMP [17]. However, others have suggested that low-dose $\alpha 2 \mathrm{AR}$ agonists reduce cAMP levels by inhibiting AC activity and vice versa. Pohjanoksa and his colleagues believe that $\alpha 2 \mathrm{AR}-$ related $\mathrm{AC}$ regulation depends on the $\alpha 2 \mathrm{AR}$ density and the activity of AC at the time [18]. Thus, it can be seen that the effect of $\alpha 2 \mathrm{AR}$ agonists on the activity of AC has a subtle dual-direction regulation effect, which is related to the physical condition $[16,18,19]$. The main molecular mechanisms governing organ protection by $\alpha 2 \mathrm{AR}$ agonists are shown in Figure 1.

\section{The Main Biological Effects of Using Dexmedetomidine for Organ Protection}

3.1. Anti-Inflammatory. In addition to the main biological effects of $\alpha 2 \mathrm{AR}$ agonists, DEX has confirmed the antiinflammatory effect of $\alpha 2 \mathrm{AR}$ agonists by reducing the inflammatory cytokine (such as TNF- $\alpha$ and IL-6) in vitro, in vivo, and in clinical experiments [20,21]. Since then, DEX's organ-protective effects as an anti-inflammatory have become a popular topic. In summary, the anti-inflammatory effects of DEX function through the following means: inhibition of TLR4/NF- $\kappa \mathrm{B}$ [13, 22-24], JAK2-STAT3 [25, 26], and $\mathrm{NF}-\kappa \mathrm{B} / \mathrm{COX}-2$ [27] pathways. DEX also promotes the release of acetylcholine $(\mathrm{ACh})$ through an antisympathetic effect; this combines with $\alpha 7 \mathrm{nAChR}$ on immune cytomembranes and exerts anti-inflammatory effects via the cholinergic pathway (as in Figure 1) [13, 26, 28].

3.2. Antiapoptotic. With the advancement of DEX research in recent years, researchers have found that DEX also has an antiapoptotic effect. The antiapoptosis function of DEX is achieved by activating the PI3K/Akt signaling pathway [29], 


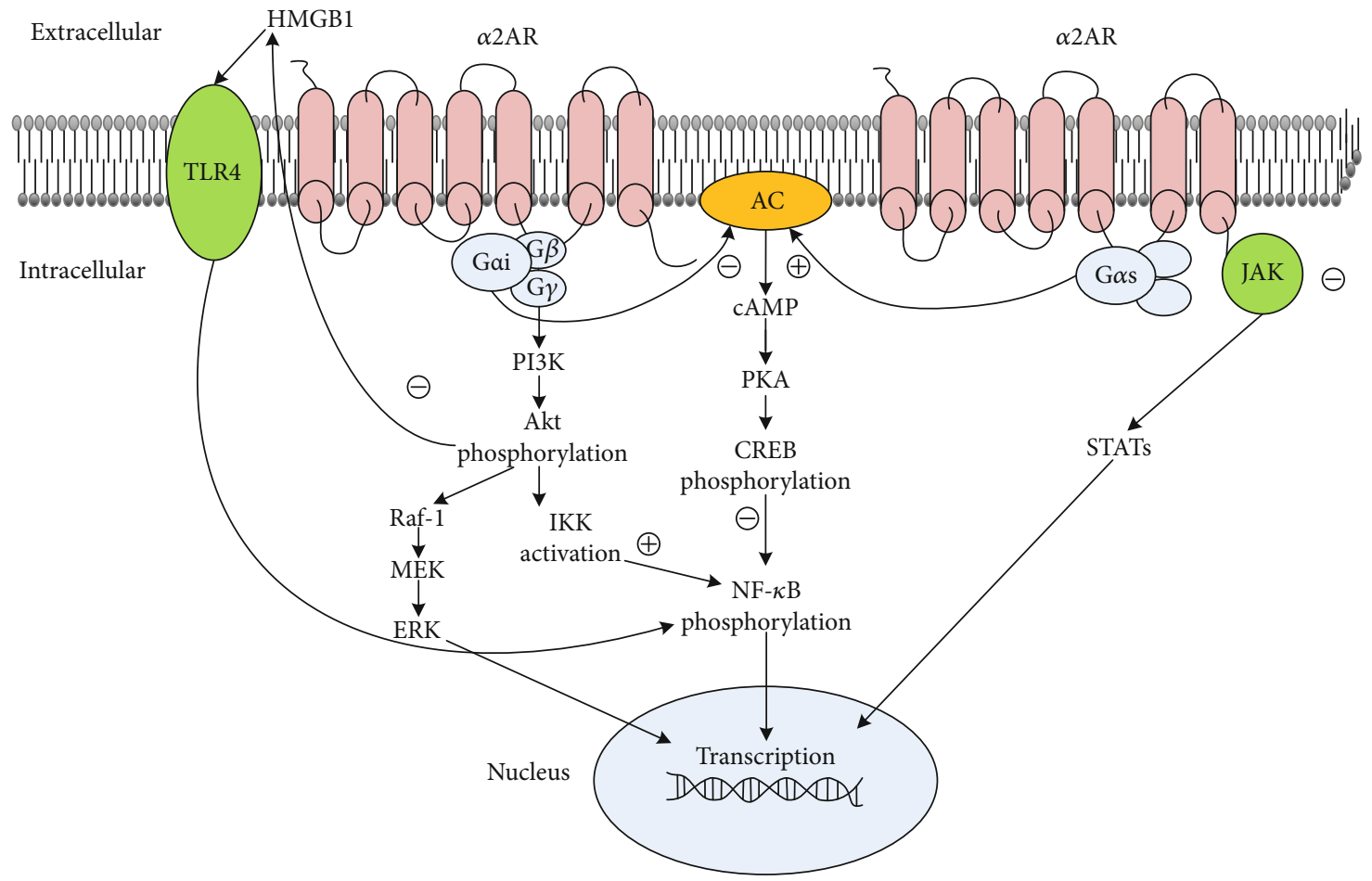

FIGURE 1: Main mechanisms of the organ-protective effects of $\alpha 2 \mathrm{AR}$ agonists. $\alpha 2 \mathrm{ARs}$ are classic inhibitory GPCRs (Gi). On the one hand, $\alpha 2$ ARs directly inhibit the AC-cAMP-PKA pathway through G $\alpha$ i proteins; on the other hand, the separation of the G $\beta \gamma$ subunit from G $\alpha \mathrm{i}$ activates the PI3K/Akt pathway and influences a series of downstream signaling including MEK/ERK, HGMB1/TLR4/NF- $\kappa \mathrm{B}$, and IKK/NF- $\kappa$ B. Incidentally, $\alpha 2 \mathrm{AR}$ agonists may inhibit JAK/STAT pathways. But when under high concentrations of an agonist and high receptor expression, $\alpha 2 \mathrm{ARs}$ may couple both physically and functionally to Gs, producing an opposite effect of inhibiting NF- $\kappa \mathrm{B}$.

the Bax/cytochrome C/caspase pathway [15], and the ERK1/2 signaling pathway $[18,30]$, as well as inhibiting Notch/NF- $\kappa$ B signaling pathways [31] and activating the mitochondrial ATP-sensitive $\mathrm{K}+$ (mitoKATP) channels (see Figure 1) [32, 33].

\section{The Mechanism of Organ-Protective Effects of Dexmedetomidine}

4.1. Nerve System Protection. In studies of the protective effects of DEX on organs, those focused on the nervous system were the earliest and deepest. Jiang et al. [34] first reported the protective effect of DEX on hypoxic-ischemic brain damage in 1991. In the past 20 years, people have mainly focused on three aspects: (1) protecting against cerebral ischemia/hypoxia injury and improving nervous system function after brain injury [35], (2) reducing the damage of anesthetics on developing neurons [36-38], and (3) decreasing the incidence of postoperative delirium or cognitive dysfunction $[39,40]$. Currently, great progress has been made in research on the effects and mechanisms of DEX, suggesting that DEX has application values in newborns, children, patients with craniocerebral injury or stroke, and elderly patients with degraded cerebral function. Going forward, more efforts will be needed to validate laboratory research in the clinic.
Regarding the protective effect of DEX on the nervous system, it has five main functions: (1) inhibiting the excitability of sympathetic nerves and regulating the release of catecholamines, (2) regulating the release of central glutamate, (3) inhibiting cell apoptosis and release of inflammatory cytokines, (4) antioxidant stress, and (5) regulating synaptic plasticity and reducing neurotoxicity of anesthetics. The inhibition of cell apoptosis and release of inflammatory cytokines are considered to be paramount.

4.1.1. Regulates the Release of Catecholamine. Cerebral ischemia/hypoxia causes the brain to superfluously release catecholamines. This causes calcium overload in neurons, generates abundant neurotoxic free radicals, aggravates ischemia and hydrocephalus through severe cerebrovascular contraction, and increases the sensitivity of neurons to glutamate, exacerbating its destructiveness [41, 42]. DEX can effectively reduce the production of catecholamine, reducing cerebral vascular spasms and subsequent brain damage $[16,35]$.

4.1.2. Inhibits the Release of Glutamate. Glutamate, an excitatory neurotransmitter released during cerebral ischemia, excites receptors and causes neuronal damage. DEX dosedependently inhibits the release of glutamate caused by multiple channels [43, 44], significantly reduces the hypoxia and depolarization-induced increase of extracellular glutamate 
[45], and reduces the accumulation of glutamate by inhibiting the absorption of neurocyte to glutamate.

\subsubsection{Anti-Inflammatory and Antiapoptotic Effects. Cerebral} ischemia, traumatic injury, and postoperative cognitive dysfunction (POCD) are closely related to neuron inflammation and apoptosis. DEX has shown its anti-inflammatory and antiapoptotic effects in multiple animal models [46-51]. As we mentioned above, the anti-inflammatory effect of DEX is achieved by inhibiting the TLR4/NF- $\kappa$ B [47], JAK2-STAT3 [25, 50], and NF- $\kappa \mathrm{B} / \mathrm{COX}-2$ pathways [27, 52]; activating the ERK1/2 pathway [53]; and releasing acetylcholine (ACh) through antisympathetic effects via the cholinergic pathway [13]. At the same time, DEX also reduces neuronal apoptosis through a variety of mechanisms that enhance the viability of the neurocyte. These include increasing the expression of antiapoptotic proteins $\mathrm{Mdm}-2$ and Bcl-2, inhibiting proapoptotic Bax and p53, reducing the permeability of mitochondrial membranes, reducing the release of cytochrome $\mathrm{C}$ and apoptosis-inducing factors into the cytoplasm [54], activating the PI3K/Akt pathway and reducing cysteinyl aspartate proteinase (caspase-3) by promoting the phosphorylation of the focal adhesion kinase (FAK) [43], enhancing the phosphorylation of ERK1/2 by inhibiting neuronal sodium ions and delaying potassium ion influx $[4,55]$, and inhibiting the Notch/NF- $\kappa$ B signaling pathway [31].

4.1.4. Antioxidant Stress. The brain is very sensitive to the destruction of oxygen-free radicals, and ischemiareperfusion injuries produce an intracorporal antioxidantperoxidation state imbalance. DEX eliminates the excessive free radicals in the body and reduces this pathological chain reaction by reducing malondialdehyde (MDA) and improving the activity of superoxide dismutase $[56,57]$.

\subsubsection{Reduces Neurotoxicity of Anesthetics and Regulates} Synaptic Plasticity. Many studies have confirmed that general anesthetics cause neurotoxicity in the developing brain, produce neural apoptosis, and inhibit the establishment of connections between synapses $[36,45]$. Three large retrospective clinical studies showed that the general anesthesia of healthy children under 2 years of age had little correlation with postoperative neurodevelopmental hypogenesis. However, the risk in premature babies and those with congenital heart disease is greatly increased [58]. Based on this, in 2016, the FDA reiterated that repeated application of general anesthetics and sedatives to children under three years old and women in the last three months of pregnancy may affect child and fetus development.

DEX reduces the neurotoxicity of sevoflurane through many pathways: inhibiting apoptosis and autophagy [59], increasing the expression of tyrosine kinase $\mathrm{B}(\mathrm{TrkB})$ and BDNF, promoting neurocyte proliferation, maintaining nervous system function [60], inhibiting neuronal mitochondrial dynamin-related protein (Drp1) [37], and dosedependently activating the bone morphogenetic protein (BMP)/Smad pathway to regulate self-renewal, differentiation, proliferation, migration, and apoptosis of the neuro- cyte [61]. The multiple signal pathways we mentioned above show that DEX has played a significant role in neuroprotection during infant development, laying the foundation for the safe adhibition of DEX in children and pregnant women and solving the problem of general anesthesia drug selection for children.

Synaptic plasticity, the property of adjustable connection between synapses, is closely related to learning and memory since it controls synaptic information transmission. DEX pretreatment significantly improved the decline of the proliferative capacity and decreased neuronal plasticity in neonatal rats after hyperoxia induction [62]. The research on regulation of synaptic plasticity through DEX has important implications for neurodevelopmental protection and promotes the study of synaptic plasticity regulation. What is more, it is a meaningful breakthrough in the treatment of neurological diseases such as memory deficits.

4.2. Cardioprotective Benefits. Intraoperative patients are susceptible to stimuli such as surgical procedures and endotracheal intubation, which cause excitation in sympathetic nerves. This can lead to tachycardia, increased blood pressure, imbalance of myocardial blood oxygen supply and demand, and cardiac complications. The main pharmacological effects of DEX are reducing the excitability of the sympathetic nervous system, weakening the stress response, and stabilizing hemodynamics. Thus, it effectively prevents the occurrence of myocardial ischemia in the perioperative period. A large number of clinical observations have confirmed that DEX reduces postoperative mortality and myocardial infarction $[63,64]$.

We conclude that the cardioprotective mechanism of DEX is mainly reflected in the following three aspects:

(1) Inhibition of norepinephrine neuron activity in the locus coeruleus, which suppresses sympathetic nerve excitation to reduce catecholamine levels in the blood, cardiac load, and myocardial oxygen consumption. At the same time, it prolongs diastolic perfusion time, increases left ventricular coronary blood flow, reduces myocardial lactic acid release, and improves myocardial resistance to ischemia and hypoxia

(2) DEX directly inhibits cardiac norepinephrine release to reduce the occurrence of arrhythmias in high-risk patients

(3) Anti-inflammation and antiapoptosis: DEX pretreatment activates some of the signal pathways through G protein like PI3K/Akt and MEK1-2-ERK1/2, sequentially reducing apoptosis and inflammatory response caused by ischemia-reperfusion and reducing the myocardial infarction area [65-67]. The downstream molecular mechanism is not completely clear at present

Through a review of laboratory and clinical research, a new perspective is gradually formed: rational use of DEX during the perioperative period may effectively reduce the risk of cardiovascular surgery $[68,69]$. However, experiments 
by Mimuro et al. [70] have questioned this. They found that during ischemia-reperfusion injury in mouse myocardium, DEX increases the myocardial infarction area without altering hemodynamics and coronary blood flow. This effect is antagonized by yohimbine, an $\alpha 2 \mathrm{AR}$ antagonist. Although such objections are in the minority, they still need to be taken seriously. Therefore, the cardioprotective effects of DEX need to be further studied and discussed.

4.3. Pulmonary Protection. The lungs, as sensitive organs, are extremely vulnerable to systemic inflammation and remote organ ischemia-reperfusion injury. Many clinical practices-such as trauma, one-lung ventilation, extracorporeal circulation, and liver transplantation-cause lung damage through inflammation and apoptosis. Ventilation-associated lung injury (VALI) is also frequently associated with acute respiratory distress syndrome (ARDS) during treatment. In recent years, it has been observed that DEX has pulmonary protection benefits in many cases of lung injuries through its effect on the pulmonary vasoconstriction mechanism, pulmonary vascular ischemia-reperfusion injury, and release of inflammatory cytokine.

4.3.1. Relieves Inflammation, Apoptosis, and Oxidative Stress from Various Pathological Injuries. In models of acute lung injury caused by renal ischemia-reperfusion injury, DEX pre- and posttreatment significantly reduce pulmonary edema and inflammatory response by reducing myeloperoxidase (MPO) activity as well as downregulating intercellular adhesion molecule-1 (ICAM-1) and TNF- $\alpha$ mRNA expression $[71,72]$. In the rat sepsis model [24], DEX inhibits inflammatory response in the lung by the TLR4/myeloid differentiation factor $88 / \mathrm{NF}-\kappa \mathrm{B}$ pathway and reduces mortality by activating PI3K/Akt/mTOR pathways. In the rat chest trauma model, DEX also has a protective effect on pulmonary contusion [73], which reduces proinflammatory cytokines by inhibiting the activity of NF- $\kappa$ B. Furthermore, DEX reduces mitochondrial dysfunction, oxidative stress, and apoptosis in LPS-induced acute lung injury [74]. Moreover, the pulmonary protection effect of DEX has a parabolic correlation with its concentration. It shows that $50 \mu \mathrm{g} / \mathrm{kg}$ is the strongest concentration: 10 and $100 \mu \mathrm{g} / \mathrm{kg}$ did have an effect, but it was weaker than at $50 \mu \mathrm{g} / \mathrm{kg}[54,75]$.

4.3.2. Reduces VALI and Hypoxic Pulmonary Vasoconstriction. On the one hand, VALI is caused by diffuse alveolocapillary membrane damage and increased permeability due to excessive airway pressure (volutrauma). On the other hand, intense mechanical stretching activates a variety of mediators involved in inflammation in the lung's inherent cells, promotes the transcription and expression of proinflammatory factors, triggers a waterfall of inflammation, and eventually leads to the occurrence of lung injury [76]. Yang et al. [77] used the high tidal volume ventilation (HVT) mode to induce lung injury in rats. When they intravenously injected ten times the clinical dose, DEX significantly improved the lung injury with respect to its pathological morphology, inflammatory cytokines, and chemokines.
In addition, DEX improves hypoxic pulmonary vasoconstriction (HPV) and oxygenation during one-lung ventilation. In an isolated lung, the imbalance of ventilator-flow ratios causes hypoxemia; in response, the body compensates for HPV by reducing the abnormal distribution of pulmonary blood flow. During anesthesia, HPV is often affected by anesthetics such as isoflurane, sevoflurane, and propofol [78]. The application of DEX indirectly affects HPV by reducing the medicinal dose of inhalation and intravenous anesthesia. At the same time [79], DEX directly enhances HPV and alters oxygenation by reducing oxidative stress and increasing nitric oxide release during one-lung ventilation.

4.4. Renal Protection. The renal blood supply accounts for $20 \%$ of cardiac output, and $90 \%-95 \%$ of this supply is distributed in the cortex. The perioperative shock, extracorporeal circulation, and usage of vasoactive drugs can easily lead to ischemia-reperfusion injury (I/R) in the renal parenchyma, especially the cortex $[26,80]$. The renoprotective effects of DEX are as follows.

4.4.1. Improves Local Renal Blood Flow and Diuresis. Ischemia-reperfusion leads to an increase in systemic and local sympathetic activity along with intense vasoconstriction in the renal cortex. DEX improves renal ischemia damage by improving outer renal medullary blood flow through local renal vasodilation [75], increasing glomerular filtration, dampening the ability of arginine vasopressin in the collecting duct, inhibiting the expression of aquaporins along with the transport of $\mathrm{Na}^{+}$and water [81], urination stimulation, and so on [82]. At the same time, DEX also reduces glomerular congestion, swelling in renal tubular epithelial cells, and luminal stenosis [83]. What is more, DEX has multiple effects during the perioperative period: in addition to analgesia, it reduces the accumulation of other analgesic and nonsteroidal anti-inflammatory drugs that increase kidney injury risk.

4.4.2. Inhibits Inflammation, Oxidative Stress, and Apoptosis. As we mentioned above, DEX blocks NF- $\kappa$ B transcription by inhibiting the TLR4/NF- $\kappa$ B and JAK/STAT pathways while activating $\alpha 7 \mathrm{nAChR}$ via the cholinergic pathway, thus producing anti-inflammatory effects $[13,26,84,85]$. As a synthetic iNOS promoter, obstruction of NF- $\kappa \mathrm{B}$ may reduce NO release, prevent oxidative stress, and reduce mitochondrial damage [86]. DEX also reduces oxidative stress by promoting the Keapl/Nrf2/ARE/HO-1 pathway [87]. Chen et al. [88] proposed that DEX exerts antiapoptotic functions by inhibiting the ROS/JNK signaling pathway, thus reducing the expression of Bax, cytochrome C, cleaved caspase-9 proteins, and cleaved caspase- 3 proteins in the mitochondrialdependent pathway.

4.4.3. Relieves Hypercoagulability. When sepsis or ischemiareperfusion injury occurs, various inflammatory cytokines stimulate endotheliocytes to release inflammatory mediators and procoagulant substances. This activates the coagulation system, leading to a hypercoagulability state. Thereafter, the diffuse microthrombus is formed in the glomerulus (which causes perirenal blood stasis), and the glomerular filtration rate decreases. The inflammatory response interacts with 
the abnormal coagulation, causing accumulation of the toxic and harmful metabolites, aggravating kidney damage, and ultimately leading to acute and chronic kidney disease. The antisympathetic effect of DEX weakens the stress response, thereby relieving the hypercoagulable state [89]. In addition, DEX can dilate blood vessels, accelerate blood flow, reduce erythrocyte aggregation, reverse blood stagnation, and reduce kidney damage caused by coagulopathy.

4.5. Hepatoprotective Effects. DEX also has hepatoprotective benefits due to its effects on inflammation, apoptosis, and oxidative stress. In animal experiments of hepatic ischemia reperfusion injury, intraperitoneal injection of DEX (10 or $100 \mu \mathrm{g} / \mathrm{kg}$ ) 30 minutes before liver ischemia can increase the levels of superoxide dismutase, catalase, and glutathione to reduce liver tissue damage [90]. In lipopolysaccharide- (LPS-) induced oxidative stress and apoptosis experiments on rat liver, it was confirmed that DEX reduces symptoms by enhancing the GSK-3 $\beta / \mathrm{MKP}-1 / \mathrm{Nrf} 2$ pathway activity through $\alpha 2 \mathrm{AR}$ [91]. Wang et al. found that DEX protects rat liver from ischemia-reperfusion injury in relation to the TLR4/NF- $\kappa$ B pathway [33]. In the LPS/D-galactosamineinduced mouse acute liver injury experiments by Yang et al., DEX inhibited the release of TNF- $\alpha$, phosphorylation of c-jun-N-terminal kinase (JNK), and cleavage of caspase-3. By reducing the activity of caspase-3, caspase- 8 , and caspase-9, it helped relieve hepatocyte apoptosis [92]. But the molecular mechanism and clinical significance of this activity require further research and clarification.

4.6. Intestinal Protection. The intestinal barriers are important for the body's resistance to external pathogens and toxins, inflammation, stress, surgical trauma, and hypovolemia; however, ischemia-reperfusion injury can cause intestinal barrier damage $[93,94]$. Thus, bacteria and toxins in the intestine can reach the mesenteric lymphoid tissues, lymph fluid, blood, and extraintestinal tissues and organs, causing gut-derived sepsis and eventually leading to multiple organ dysfunction syndrome (MODS) [95]. Attention to the protection of intestinal barrier functions under pathological conditions can reduce the incidence of gut-derived sepsis, improve the prognosis of patients, and reduce the mortality of critical patients. Therefore, in recent years, the protection of intestinal health during the perioperative period has been increasingly recognized.

4.6.1. Anti-Inflammation, Antioxidative Stress, and Antiapoptosis. The effects of DEX against inflammation, apoptosis, and oxidative stress that we have described above also function in intestinal protection. Zhang et al. [54] studied intestinal ischemia-reperfusion injuries in rats, administering DEX at different times and doses. They showed that DEX pretreatment was dose-dependent for resisting intestinal ischemia-reperfusion injury in a certain range. Their experiments suggested that in addition to the reduction of oxidative stress, inflammation indicators of malondialdehyde (MDA) and myeloperoxidase (MPO), serum levels of diamine oxidase (DAO), activity of caspase-3, ileal mucosa levels, and the apoptotic index of ileal mucosal cells were significantly lower than in those rats without DEX. It was shown that the effect of DEX is maximal at $5 \mu \mathrm{g} / \mathrm{kg} / \mathrm{h}$, but has no effect in posttreatment. The mechanism of the difference in preand posttreatment needs further research.

In addition, some clinical trials have also highlighted the intestinal protective effect of DEX. DEX pretreatment relieves intestinal and liver damage in patients undergoing liver cancer resection who are afflicted by selective hepatic lobectomy and liver cirrhosis [96]. DEX also has significant preventive effects on postoperative abdominal adhesions [97], and its mechanism may relate to antioxidative stress effects and reduction of tissue inflammation.

4.6.2. Promotes Recovery of Intestinal Motility, Improves Intestinal Microcirculation, and Protects against Intestinal Epithelial Barrier Disruption. DEX promotes isolated rat ileum contraction [98]. In contrast, Herbert et al. [99] found that DEX dose-dependently inhibited the enterocinesia of guinea pigs' small intestines in vitro. In healthy volunteers, DEX also inhibits gastric emptying and enterocinesia [100]. These contradictory results may be related to the physiological state of the different subjects. Under normal physiological conditions, DEX may inhibit enterogastric peristalsis via enteric neurons. On the other hand, under pathological conditions such as infection, stress, trauma, and hypovolemia, DEX benefits intestinal microcirculation perfusion, protects the gut barrier, and restores gastrointestinal motility. It does the latter by reducing inflammation and stress reactions, maintaining hemodynamic stability, relieving postoperative pain, and reducing the dosage of postoperative opioids. In rat models, DEX protects against intestinal epithelial barrier disruption by recovering the density of small blood vessels perfused in the intestinal mucosa and muscles, attenuating intestinal microcirculatory dysfunction, and inhibiting inflammatory response, thus reducing mucosal cell death and tight junctional damage $[56,101]$.

\section{Correlation of Anti-Inflammatory Effects with Timing and Dose}

The anti-inflammatory effect of DEX has some correlation with its dose. In sepsis rats, Taniguchi et al. [102] found that the medium dose $(5 \mu \mathrm{g} / \mathrm{kg} / \mathrm{h})$ and high dose $(10 \mu \mathrm{g} / \mathrm{k} / \mathrm{h})$ of DEX reduce plasma levels of TNF- $\alpha$ and IL-6, while the low-dose group $(2.5 \mu \mathrm{g} / \mathrm{kg} / \mathrm{h})$ showed no decreasing effect. Similar experimental phenomena were observed in vitro $[43,103]$. Lai et al. [104] believed that the anti-inflammatory effect of DEX had a parabolic relationship with its concentration. In their in vitro experiment, LPS-induced expression of inflammatory cytokines was treated with different concentrations of DEX. The result shows that while the addition of $0.01 \mu \mathrm{mol} / \mathrm{L}$ of DEX had no impact, and the addition of $1 \mu \mathrm{mol} / \mathrm{L}$ of DEX was significantly inhibited, $100 \mu \mathrm{mol} / \mathrm{L}$ of DEX promoted the expression of nitric oxide synthase and nitric oxide synthesis; the effects of inflammatory cytokines such as prostaglandin E2, TNF- $\alpha$, IL- $1 \beta$, IL-6, and IL-10 are consistent with the effects of nitric oxide. Some other scholars have also observed the same trend $[54,55]$. 
In addition, $\mathrm{Gu}$ et al. believe that the protective effect of DEX on organs is related to the processing time, and the posttreatment conditioning is less than the pretreatment [11]. Zhang et al. believe that DEX posttreatment does not alleviate dilated reperfusion injury in the small intestine [54]. Based on the physiological characteristics of $\alpha 2 \mathrm{AR}$ described above, $\alpha 2 \mathrm{AR}$-related AC regulation is affected by factors such as $\alpha 2 \mathrm{AR}$ density and AC activity at the time, while the latter two are related to the state of the body. So we speculate that there are two situations: (1) $\alpha 2 \mathrm{AR}$ density and $\mathrm{AC}$ activity may be affected by different stages of inflammation in the body or organs; (2) although DEX is mainly coupled with the Gi protein, in a certain concentration range, it may also be coupled with the Gs protein. The latter leads to a subtle two-way regulation effect on AC activity and even the downstream pathways. As a result, some scholars have observed dose-dependent changes in organ protection and differential results in pre- and posttreatment. Consequently, in the future, it is necessary to search for a more safe and effective concentration range and medication timing in the usage of DEX.

\section{Perspective}

In conclusion, the organ-protective effect of DEX is achieved in different pathological conditions by multiple mechanisms. However, a majority of the current DEX studies are based on animal experiments, and the mechanism is not fully elucidated. More intensive research and clinical trials are necessary for further verification. In addition, despite the mainstream belief that the organ-protective effect of DEX seems clear, there are conflicting conclusions about whether $\alpha 2 \mathrm{AR}$ agonism is protective or destructive. Therefore, while considering the sedative, analgesic, and organ-protective effects of DEX, we need to explore the different rates of timing, appropriate dosages, and medication profiles of this substance. This will help to promote realistic, significant clinical observations that validate the potential for using DEX in the future to protect organs.

\section{Conflicts of Interest}

The authors declare that there is no conflict of interest regarding the publication of this article.

\section{Acknowledgments}

This work was supported by the Science and Technology Research Project of the Liaoning Provincial Education Department (LK201651).

\section{References}

[1] A. Paris and P. H. Tonner, "Dexmedetomidine in anaesthesia," Current Opinion in Anaesthesiology, vol. 18, no. 4, pp. 412-418, 2005.

[2] A. J. Lewington, J. Cerda, and R. L. Mehta, "Raising awareness of acute kidney injury: a global perspective of a silent killer," Kidney International, vol. 84, no. 3, pp. 457-467, 2013.
[3] E. F. Daher, G. B. Silva Junior, S. Q. Santos et al., "Differences in community, hospital and intensive care unit-acquired acute kidney injury: observational study in a nephrology service of a developing country," Clinical Nephrology, vol. 78, no. 6, pp. 449-455, 2012.

[4] T. Kamibayashi and M. Maze, "Clinical uses of alpha2adrenergic agonists," Anesthesiology, vol. 93, no. 5, pp. 1345-1349, 2000.

[5] M. Maze, M. Maze, and M. Fujinaga, "Alpha 2 adrenoceptors in pain modulation: which subtype should be targeted to produce analgesia?," Anesthesiology, vol. 92, no. 4, pp. 934-936, 2000.

[6] T. Taniguchi, Y. Kidani, H. Kanakura, Y. Takemoto, and K. Yamamoto, "Effects of dexmedetomidine on mortality rate and inflammatory responses to endotoxin-induced shock in rats," Critical Care Medicine, vol. 32, no. 6, pp. 1322-1326, 2004.

[7] M. I. Simon, M. P. Strathmann, and N. Gautam, "Diversity of G proteins in signal transduction," Science, vol. 252, no. 5007, pp. 802-808, 1991.

[8] K. K. Meldrum, K. Hile, D. R. Meldrum, J. A. Crone, J. P. Gearhart, and A. L. Burnett, "Simulated ischemia induces renal tubular cell apoptosis through a nuclear factor-kappaB dependent mechanism," The Journal of Urology, vol. 168, no. 1, pp. 248-252, 2002.

[9] K. DONNAHOO, D. R. MELDRUM, R. SHENKAR, C. S. CHUNG, E. ABRAHAM, and A. H. HARKEN, "Early renal ischemia, with or without reperfusion, activates NFkappaB and increases TNF-alpha bioactivity in the kidney," The Journal of Urology, vol. 163, no. 4, pp. 1328-1332, 2000.

[10] M. S. Hayden and S. Ghosh, "Signaling to NF-kappaB," Genes \& Development, vol. 18, no. 18, pp. 2195-2224, 2004.

[11] X. Y. Gu, B. L. Liu, K. K. Zang et al., "Dexmedetomidine inhibits tetrodotoxin-resistant Nav1.8 sodium channel activity through Gi/o-dependent pathway in rat dorsal root ganglion neurons.," Molecular Brain, no. 1, article S13041015-0105-2, p. 15, 2015.

[12] H. Rong, Z. Zhao, J. Feng et al., "The effects of dexmedetomidine pretreatment on the pro- and anti-inflammation systems after spinal cord injury in rats," Brain, Behavior, and Immunity, vol. 64, article S0889159117300685, pp. 195-207, 2017.

[13] J. Gu, P. Sun, H. Zhao et al., "Dexmedetomidine provides renoprotection against ischemia-reperfusion injury in mice," Critical Care, vol. 15, no. 3, p. R153, 2011.

[14] B. Li, T. du, H. Li et al., "Signalling pathways for transactivation by dexmedetomidine of epidermal growth factor receptors in astrocytes and its paracrine effect on neurons," British Journal of Pharmacology, vol. 154, no. 1, pp. 191203, 2008.

[15] M. G. Eason, H. Kurose, B. D. Holt, J. R. Raymond, and S. B. Liggett, "Simultaneous coupling of alpha 2-adrenergic receptors to two G-proteins with opposing effects. Subtypeselective coupling of alpha $2 \mathrm{C} 10$, alpha $2 \mathrm{C} 4$, and alpha $2 \mathrm{C} 2$ adrenergic receptors to $\mathrm{Gi}$ and Gs," The Journal of Biological Chemistry, vol. 267, no. 22, pp. 15795-15801, 1992.

[16] C. M. Fraser, S. Arakawa, W. McCombie, and J. C. Venter, "Cloning, sequence analysis, and permanent expression of a human alpha 2-adrenergic receptor in Chinese hamster ovary cells. Evidence for independent pathways of receptor coupling to adenylate cyclase attenuation and activation," The 
Journal of Biological Chemistry, vol. 264, no. 20, pp. 1175411761, 1989.

[17] M. A. Daemen, B. de Vries, and W. A. Buurman, "Apoptosis and inflammation in renal reperfusion injury," Transplantation, vol. 73, no. 11, pp. 1693-1700, 2002.

[18] K. Pohjanoksa, C. . Jansson, K. Luomala, A. Marjamäki, J. M. Savola, and M. Scheinin, " $\alpha 2$-Adrenoceptor regulation of adenylyl cyclase in $\mathrm{CHO}$ cells: Dependence on receptor density, receptor subtype and current activity of adenylyl cyclase," European Journal of Pharmacology, vol. 335, no. 1, article S0014299997011540, pp. 53-63, 1997.

[19] A. B. Sanz, M. D. Sanchez-Niño, A. M. Ramos et al., "NF- $\kappa B$ in renal inflammation," Journal of the American Society of Nephrology, vol. 21, no. 8, pp. 1254-1262, 2010.

[20] S. Hofer, J. Steppan, T. Wagner et al., "Central sympatholytics prolong survival in experimental sepsis," Critical Care, vol. 13, no. 1, p. R11, 2009.

[21] H. Qiao, R. D. Sanders, D. Ma, X. Wu, and M. Maze, "Sedation improves early outcome in severely septic Sprague Dawley rats," Critical Care, vol. 13, no. 4, article cc8012, p. R136, 2009.

[22] A. S. Levey and M. T. James, "Acute kidney injury," Annals of Internal Medicine, vol. 167, no. 9, pp. ITC66-ITC80, 2017.

[23] L. Yang, G. Xing, L. Wang et al., "Acute kidney injury in China: a cross-sectional survey," Lancet, vol. 386, no. 10002, article S014067361500344X, pp. 1465-1471, 2015.

[24] N. H. Lameire, A. Bagga, D. Cruz et al., "Acute kidney injury: an increasing global concern," Lancet, vol. 382, no. 9887, pp. 170-179, 2013.

[25] Y. R. Lankadeva, S. Ma, N. Iguchi et al., "Dexmedetomidine reduces norepinephrine requirements and preserves renal oxygenation and function in ovine septic acute kidney injury," Kidney International, vol. 96, no. 5, pp. 1150-1161, 2019.

[26] Y. Si, H. Bao, L. Han et al., "Dexmedetomidine protects against renal ischemia and reperfusion injury by inhibiting the JAK/STAT signaling activation," Journal of Translational Medicine, vol. 11, no. 1, article 1479-5876-11-141, p. 141, 2013.

[27] H. Yao, X. Chi, Y. Jin et al., "Dexmedetomidine Inhibits TLR4/NF- $\kappa$ B Activation and Reduces Acute Kidney Injury after Orthotopic Autologous Liver Transplantation in Rats," Scientific Reports, vol. 5, no. 1, article BFsrep16849, 2015.

[28] M. Miksa, P. Das, M. Zhou et al., "Pivotal role of the alpha (2A)-adrenoceptor in producing inflammation and organ injury in a rat model of sepsis," PLoS One, vol. 4, no. 5, article e5504, 2009.

[29] R. D. Ye, "Regulation of nuclear factor kappaB activation by G-protein-coupled receptors," Journal of Leukocyte Biology, vol. 70, no. 6, pp. 839-848, 2001.

[30] Y. M. Zhu, C. C. Wang, L. Chen et al., "Both PI3K/Akt and ERK1/2 pathways participate in the protection by dexmedetomidine against transient focal cerebral ischemia/reperfusion injury in rats," Brain Research, vol. 1494, pp. 1-8, 2013.

[31] M. S. Paller, J. R. Hoidal, and T. F. Ferris, "Oxygen free radicals in ischemic acute renal failure in the rat," The Journal of Clinical Investigation, vol. 74, no. 4, pp. 11561164, 1984.

[32] X. Chi, X. Wei, W. Gao et al., "Dexmedetomidine ameliorates acute lung injury following orthotopic autologous liver transplantation in rats probably by inhibiting toll-like recep- tor 4-nuclear factor kappa B signaling," Journal of Translational Medicine, vol. 13, no. 1, article 554, 2015.

[33] Y. Wang, S. Wu, X. Yu et al., "Dexmedetomidine Protects Rat Liver against Ischemia-Reperfusion Injury Partly by the $\alpha 2 \mathrm{~A}$ Adrenoceptor Subtype and the Mechanism Is Associated with the TLR4/NF- $\kappa$ B Pathway," International Journal of Molecular Sciences, vol. 17, no. 7, article ijms17070995, p. 995, 2016.

[34] S. Jiang, J. Streeter, B. M. Schickling, K. Zimmerman, R. M. Weiss, and Miller FJ Jr, "Nox1 NADPH oxidase is necessary for late but not early myocardial ischaemic preconditioning," Cardiovascular Research, vol. 102, no. 1, pp. 79-87, 2014.

[35] U. Kunter, S. Daniel, M. B. Arvelo et al., "Combined expression of A1 and A20 achieves optimal protection of renal proximal tubular epithelial cells," Kidney International, vol. 68, no. 4, pp. 1520-1532, 2005.

[36] Y. T. Xuan, X. L. Tang, S. Banerjee et al., "Nuclear factorkappaB plays an essential role in the late phase of ischemic preconditioning in conscious rabbits," Circulation Research, vol. 84, no. 9, pp. 1095-1109, 1999.

[37] Y. Shan, S. Sun, F. Yang, N. Shang, and H. Liu, "Dexmedetomidine protects the developing rat brain against the neurotoxicity wrought by sevoflurane: role of autophagy and Drp1-Bax signaling," Drug Design, Development and Therapy, vol. 12, pp. 3617-3624, 2018.

[38] J. Geng, Y. Liu, Y. Guo et al., "Correlation between TERT $\mathrm{C} 228 \mathrm{~T}$ and clinic-pathological features in pediatric papillary thyroid carcinoma," Science China. Life Sciences, vol. 62, no. 12, article 9546, pp. 1563-1571, 2019.

[39] A. Iida, H. Yoshidome, T. Shida et al., "Hepatocyte nuclear factor-kappa beta (NF-kappaB) activation is protective but is decreased in the cholestatic liver with endotoxemia," Surgery, vol. 148, no. 3, pp. 477-489, 2010.

[40] B. Dawn, Y. Guo, A. Rezazadeh et al., “Tumor necrosis factoralpha does not modulate ischemia/reperfusion injury in naïve myocardium but is essential for the development of late preconditioning," Journal of Molecular and Cellular Cardiology, vol. 37, no. 1, pp. 51-61, 2004.

[41] J. Lutz, L. A. Luong, M. Strobl et al., "The A20 gene protects kidneys from ischaemia/reperfusion injury by suppressing pro-inflammatory activation," Journal of Molecular Medicine (Berlin, Germany), vol. 86, no. 12, article 405, pp. 1329-1339, 2008.

[42] C. G. da Silva, E. R. Maccariello, S. W. Wilson et al., "Hepatocyte growth factor preferentially activates the anti-inflammatory arm of NF- $\kappa$ B signaling to induce A20 and protect renal proximal tubular epithelial cells from inflammation," Journal of Cellular Physiology, vol. 227, no. 4, pp. 1382-1390, 2012.

[43] S. Bunte, F. Behmenburg, N. Majewski et al., "Characteristics of dexmedetomidine postconditioning in the field of myocardial ischemia-reperfusion injury," Anesthesia and Analgesia, vol. 130, no. 1, pp. 90-98, 2020.

[44] K. Nishina, H. Akamatsu, K. Mikawa et al., "The effects of clonidine and dexmedetomidine on human neutrophil functions," Anesthesia and Analgesia, vol. 88, no. 2, pp. 452-458, 1999.

[45] D. J. Pepperl and J. W. Regan, "Selective coupling of alpha 2adrenergic receptor subtypes to cyclic AMP-dependent reporter gene expression in transiently transfected JEG-3 cells," Molecular Pharmacology, vol. 44, no. 4, pp. 802-809, 1993. 
[46] D. M. Rosenbaum, S. G. Rasmussen, and B. K. Kobilka, "The structure and function of G-protein-coupled receptors," Nature, vol. 459, no. 7245, pp. 356-363, 2009.

[47] A. V. Smrcka and I. Fisher, "G-protein $\beta \gamma$ subunits as multifunctional scaffolds and transducers in G-protein-coupled receptor signaling," Cellular and Molecular Life Sciences, vol. 76, no. 22, article 3275, pp. 4447-4459, 2019.

[48] K. A. Meller, J. Całka, M. Kaczmarek, and B. Jana, "Expression of alpha and beta adrenergic receptors in the pig uterus during inflammation," Theriogenology, vol. 119, pp. 96-104, 2018.

[49] D. Ma, N. Rajakumaraswamy, and M. Maze, " $\alpha 2$-Adrenoceptor agonists: shedding light on neuroprotection?," British Medical Bulletin, vol. 71, no. 1, pp. 77-92, 2005.

[50] H. Lavon, P. Matzner, A. Benbenishty et al., "Dexmedetomidine promotes metastasis in rodent models of breast, lung, and colon cancers," British Journal of Anaesthesia, vol. 120, no. 1, pp. 188-196, 2018.

[51] W. X. Wang, Q. Wu, S. S. Liang et al., "Dexmedetomidine promotes the recovery of neurogenesis in aged mouse with postoperative cognitive dysfunction," Neuroscience Letters, vol. 677, pp. 110-116, 2018.

[52] N. Bao, B. Tang, and J. Wang, "Dexmedetomidine preconditioning protects rats from renal ischemia-reperfusion injury accompanied with biphasic changes of nuclear factor-kappa B signaling," Journal of Immunology Research, vol. 2020, Article ID 3230490, 12 pages, 2020.

[53] H. de Castro Abrantes, M. Briquet, C. Schmuziger et al., "The lactate receptor HCAR1 modulates neuronal network activity through the activation of $\mathrm{G} \alpha$ and $\mathrm{G} \beta \gamma$ subunits," The Journal of Neuroscience, vol. 39, no. 23, pp. 4422-4433, 2019.

[54] X. Y. Zhang, Z. M. Liu, S. H. Wen et al., "Dexmedetomidine administration before, but not after, ischemia attenuates intestinal injury induced by intestinal ischemia-reperfusion in rats," Anesthesiology, vol. 116, no. 5, pp. 1035-1046, 2012.

[55] V. Hanci, G. Yurdakan, S. Yurtlu, I. Ö. Turan, and E. Y. Sipahi, "Protective effect of dexmedetomidine in a rat model of $\alpha$-naphthylthiourea-induced acute lung injury," The Journal of Surgical Research, vol. 178, no. 1, pp. 424-430, 2012.

[56] Y. C. Yeh, C. Y. Wu, Y. J. Cheng et al., "Effects of dexmedetomidine on intestinal microcirculation and intestinal epithelial barrier in endotoxemic rats," Anesthesiology, vol. 125, no. 2, pp. 355-367, 2016.

[57] Y. Li and S. Liu, "The effect of dexmedetomidine on oxidative stress response following cerebral ischemia-reperfusion in rats and the expression of intracellular adhesion molecule-1 (ICAM-1) and S100B," Medical Science Monitor, vol. 23, pp. 867-873, 2017.

[58] M. R. Graham, "Clinical update regarding general anesthesiaassociated neurotoxicity in infants and children," Current Opinion in Anaesthesiology, vol. 30, no. 6, pp. 682-687, 2017.

[59] J. F. Perez-Zoghbi, W. Zhu, M. R. Grafe, and A. M. Brambrink, "Dexmedetomidine-mediated neuroprotection against sevoflurane-induced neurotoxicity extends to several brain regions in neonatal rats," British Journal of Anaesthesia, vol. 119, no. 3, pp. 506-516, 2017.

[60] L. J. Bo, P. X. Yu, F. Z. Zhang, and Z. M. Dong, "Dexmedetomidine mitigates sevoflurane-induced cell cycle arrest in hippocampus," Journal of Anesthesia, vol. 32, no. 5, pp. 717-724, 2018.
[61] Y. Shan, F. Yang, Z. Tang et al., "Dexmedetomidine ameliorates the neurotoxicity of sevoflurane on the immature brain through the BMP/SMAD signaling pathway," Frontiers in Neuroscience, vol. 12, p. 964, 2018.

[62] S. Endesfelder, H. Makki, C. von Haefen, C. D. Spies, C. Bührer, and M. Sifringer, "Neuroprotective effects of dexmedetomidine against hyperoxia-induced injury in the developing rat brain," PLoS One, vol. 12, no. 2, article e0171498, 2017.

[63] D. N. Wijeysundera, J. S. Naik, and W. S. Beattie, “Alpha-2 adrenergic agonists to prevent perioperative cardiovascular complications: a meta-analysis," The American Journal of Medicine, vol. 114, no. 9, pp. 742-752, 2003.

[64] B. M. Biccard, S. Goga, and J. de Beurs, "Dexmedetomidine and cardiac protection for non-cardiac surgery: a metaanalysis of randomised controlled trials," Anaesthesia, vol. 63, no. 1, pp. 4-14, 2008.

[65] S. Sulaiman, R. B. Karthekeyan, M. Vakamudi, A. S. Sundar, H. Ravullapalli, and R. Gandham, "The effects of dexmedetomidine on attenuation of stress response to endotracheal intubation in patients undergoing elective off-pump coronary artery bypass grafting," Annals of Cardiac Anaesthesia, vol. 15, no. 1, pp. 39-43, 2012.

[66] M. Ibacache, G. Sanchez, Z. Pedrozo et al., "Dexmedetomidine preconditioning activates pro-survival kinases and attenuates regional ischemia/reperfusion injury in rat heart," Biochimica et Biophysica Acta, vol. 1822, no. 4, article S0925443911002985, pp. 537-545, 2012.

[67] W. Li, J. Yu, Z. Li, and W. B. Yin, "Rational design for fungal laccase production in the model host Aspergillus nidulans," Science China. Life Sciences, vol. 62, no. 1, pp. 84-94, 2019.

[68] G. Wang, J. Niu, Z. Li, H. Lv, and H. Cai, "The efficacy and safety of dexmedetomidine in cardiac surgery patients: a systematic review and meta-analysis," PLoS One, vol. 13, no. 9, article e0202620, 2018.

[69] D. Duncan, A. Sankar, W. S. Beattie, D. N. Wijeysundera, and Cochrane Anaesthesia Group, "Alpha-2 adrenergic agonists for the prevention of cardiac complications among adults undergoing surgery," Cochrane Database of Systematic Reviews, 2018.

[70] S. Mimuro, T. Katoh, A. Suzuki et al., "Deterioration of myocardial injury due to dexmedetomidine administration after myocardial ischaemia," Resuscitation, vol. 81, no. 12, pp. 1714-1717, 2010.

[71] J. Gu, J. Chen, P. Xia, G. Tao, H. Zhao, and D. Ma, "Dexmedetomidine attenuates remote lung injury induced by renal ischemia-reperfusion in mice," Acta Anaesthesiologica Scandinavica, vol. 55, no. 10, pp. 1272-1278, 2011.

[72] N. Bao and D. Dai, "Dexmedetomidine protects against ischemia and reperfusion-induced kidney injury in rats," Mediators of Inflammation, vol. 2020, Article ID 2120971, 8 pages, 2020.

[73] X. Wu, X. Song, N. Li, L. Zhan, Q. Meng, and Z. Xia, "Protective effects of dexmedetomidine on blunt chest traumainduced pulmonary contusion in rats," Journal of Trauma and Acute Care Surgery, vol. 74, no. 2, pp. 524-530, 2013.

[74] C. Fu, X. Dai, Y. Yang, M. Lin, Y. Cai, and S. Cai, "Dexmedetomidine attenuates lipopolysaccharide-induced acute lung injury by inhibiting oxidative stress, mitochondrial dysfunction and apoptosis in rats," Molecular Medicine Reports, vol. 15, no. 1, pp. 131-138, 2017. 
[75] F. T. Billings, S. W. C. Chen, M. Kim et al., " $\alpha 2$-Adrenergic agonists protect against radiocontrast-induced nephropathy in mice," American Journal of Physiology-Renal Physiology, vol. 295, no. 3, pp. F741-F748, 2008.

[76] L. I. L. I. JIANG, L. LI, J. SHEN, Z. QI, and L. GUO, "Effect of dexmedetomidine on lung ischemia-reperfusion injury," Molecular Medicine Reports, vol. 9, no. 2, pp. 419-426, 2014.

[77] C. L. Yang, P. S. Tsai, and C. J. Huang, "Effects of dexmedetomidine on regulating pulmonary inflammation in a rat model of ventilator-induced lung injury," Acta Anaesthesiologica Taiwanica, vol. 46, no. 4, pp. 151-159, 2008.

[78] R. Xia, J. Xu, H. Yin et al., "Intravenous Infusion of Dexmedetomidine Combined Isoflurane Inhalation Reduces Oxidative Stress and Potentiates Hypoxia Pulmonary Vasoconstriction during One-Lung Ventilation in Patients," Mediators of Inflammation, vol. 2015, Article ID 238041, 7 pages, 2015.

[79] E. Erturk, S. Topaloglu, D. Dohman et al., "The Comparison of the Effects of Sevoflurane Inhalation Anesthesia and Intravenous Propofol Anesthesia on Oxidative Stress in One Lung Ventilation," BioMed Research International, vol. 2014, Article ID 360936, 4 pages, 2014.

[80] S. Andonian, T. Coulthard, A. D. Smith, P. S. Singhal, and B. R. Lee, "Real-time quantitation of renal ischemia using targeted microbubbles: in-vivo measurement of P-selectin expression," Journal of Endourology, vol. 23, no. 3, pp. 373378, 2009.

[81] A. J. Rouch, L. H. Kudo, and C. Hebert, "Dexmedetomidine inhibits osmotic water permeability in the rat cortical collecting duct," The Journal of Pharmacology and Experimental Therapeutics, vol. 281, no. 1, pp. 62-69, 1997.

[82] A. Junaid, L. Cui, S. B. Penner, and D. D. Smyth, "Regulation of aquaporin-2 expression by the alpha (2)-adrenoceptor agonist clonidine in the rat," The Journal of Pharmacology and Experimental Therapeutics, vol. 291, no. 2, pp. 920-923, 1999.

[83] M. Cakir, A. Polat, S. Tekin et al., "The effect of dexmedetomidine against oxidative and tubular damage induced by renal ischemia reperfusion in rats," Renal Failure, vol. 37, no. 4, pp. 704-708, 2015.

[84] P. K. Chatterjee, Y. al-Abed, B. Sherry, and C. N. Metz, "Cholinergic agonists regulate JAK2/STAT3 signaling to suppress endothelial cell activation," American Journal of Physiology. Cell Physiology, vol. 297, no. 5, pp. C1294-C1306, 2009.

[85] W. J. de Jonge, E. van der Zanden, F. O. The et al., "Stimulation of the vagus nerve attenuates macrophage activation by activating the Jak 2-STAT3 signaling pathway," Nature Immunology, vol. 6, no. 8, pp. 844-851, 2005.

[86] Y. J. Ji, H. N. Liu, X. F. Kong et al., "Use of insect powder as a source of dietary protein in early-weaned piglets1," Journal of Animal Science, vol. 94, suppl_3, pp. 111-116, 2016.

[87] X. Yu, X. Chi, S. Wu et al., "Dexmedetomidine Pretreatment Attenuates Kidney Injury and Oxidative Stress during Orthotopic Autologous Liver Transplantation in Rats," Oxidative Medicine and Cellular Longevity, vol. 2016, Article ID 4675817, 10 pages, 2016.

[88] Y. Chen, X. Feng, X. Hu et al., "Dexmedetomidine Ameliorates Acute Stress-Induced Kidney Injury by Attenuating Oxidative Stress and Apoptosis through Inhibition of the ROS/JNK Signaling Pathway," Oxidative Medicine and Cellular Longevity, vol. 2018, Article ID 4035310, 12 pages, 2018.
[89] Z. Chen, D. H. Shao, Z. M. Mao, L. L. Shi, X. D. Ma, and D. P. Zhang, "Effect of dexmedetomidine on blood coagulation in patients undergoing radical gastrectomy under general anesthesia: a prospective, randomized controlled clinical trial," Medicine (Baltimore), vol. 97, no. 27, article e11444, 2018.

[90] T. Sahin, Z. Begeç, H. İ. Toprak et al., "The effects of dexmedetomidine on liver ischemia-reperfusion injury in rats," The Journal of Surgical Research, vol. 183, no. 1, pp. 385-390, 2013.

[91] J. Sha, H. Zhang, Y. Zhao et al., "Dexmedetomidine attenuates lipopolysaccharide-induced liver oxidative stress and cell apoptosis in rats by increasing GSK-3 drenergic agonists protect aga MKP-1/Nrf2 pathway activity via the $\alpha 2$ adrenergic receptor," Toxicology and Applied Pharmacology, vol. 364, article S0041008X18305763, pp. 144-152, 2019.

[92] C. Yang, L. He, C. Wang et al., "Dexmedetomidine alleviated lipopolysaccharide/D-galactosamine-induced acute liver injury in mice," International Immunopharmacology, vol. 72, pp. 367-373, 2019.

[93] K. Wang, Z. Wan, A. Ou et al., "Monofloral honey from a medical plant, Prunella vulgaris, protected against dextran sulfate sodium-induced ulcerative colitis via modulating gut microbial populations in rats," Food \& Function, vol. 10, no. 7, pp. 3828-3838, 2019.

[94] K. Wang, X. Jin, Q. Li et al., "Propolis from Different Geographic Origins Decreases Intestinal Inflammation andBacteroidesspp. Populations in a Model of DSS-Induced Colitis," Molecular Nutrition \& Food Research, vol. 62, no. 17, article 1800080, 2018.

[95] R. P. Dickson, "The microbiome and critical illness," The Lancet Respiratory Medicine, vol. 4, no. 1, article S2213260015004270, pp. 59-72, 2016.

[96] Z. X. Wang, C. Y. Huang, Y. P. Hua, W. Q. Huang, L. H. Deng, and K. X. Liu, "Dexmedetomidine reduces intestinal and hepatic injury after hepatectomy with inflow occlusion under general anaesthesia: a randomized controlled trial," British Journal of Anaesthesia, vol. 112, no. 6, pp. 10551064, 2014.

[97] S. Kuru, O. B. Bozkirli, A. M. Barlas et al., "The preventive effect of dexmedetomidine against postoperative intraabdominal adhesions in rats," International Surgery, vol. 100, no. 1, pp. 87-95, 2015.

[98] C. Aydin, I. Bagcivan, S. Gursoy, A. Altun, O. Topcu, and A. Koyuncu, "Altered spontaneous contractions of the ileum by anesthetic agents in rats exposed to peritonitis," World Journal of Gastroenterology, vol. 15, no. 13, pp. 1620-1624, 2009.

[99] M. K. Herbert, S. Roth-Goldbrunner, P. Holzer, and N. Roewer, "Clonidine and dexmedetomidine potently inhibit peristalsis in the guinea pig ileum in vitro," Anesthesiology, vol. 97, no. 6, pp. 1491-1499, 2002.

[100] T. Iirola, S. Vilo, R. Aantaa et al., "Dexmedetomidine inhibits gastric emptying and oro-caecal transit in healthy volunteers," British Journal of Anaesthesia, vol. 106, no. 4, pp. 522-527, 2011.

[101] Y.-C. Yeh, W.-Z. Sun, W.-J. Ko et al., "Dexmedetomidine Prevents Alterations of Intestinal Microcirculation That Are Induced by Surgical Stress and Pain in a Novel Rat Model," Anesthesia and Analgesia, vol. 115, no. 1, pp. 46-53, 2012.

[102] T. Taniguchi, A. Kurita, K. Kobayashi, K. Yamamoto, and H. Inaba, "Dose- and time-related effects of dexmedetomidine on mortality and inflammatory responses to 
endotoxin-induced shock in rats," Journal of Anesthesia, vol. 22, no. 3, pp. 221-228, 2008.

[103] C. Xie, Z. Wang, J. Tang, Z. Shi, and Z. He, "The effect of dexmedetomidine post-treatment on the inflammatory response of astrocyte induced by lipopolysaccharide," Cell Biochemistry and Biophysics, vol. 71, no. 1, pp. 407-412, 2015.

[104] Y. C. Lai, P. S. Tsai, and C. J. Huang, "Effects of Dexmedetomidine on Regulating Endotoxin-Induced Up-Regulation of Inflammatory Molecules in Murine Macrophages," The Journal of Surgical Research, vol. 154, no. 2, article S0022480408004654, pp. 212-219, 2009. 